\title{
Sala de Estudos Aumentada: Suporte Computacional para o Desenvolvimento Técnico e Perceptivo da Performance Musical
}

\author{
Aluizio Barbosa de Oliveira Neto ${ }^{1}$, Mauricio Alves Loureiro ${ }^{2}$ \\ ${ }^{1}$ Informática e Comunicação - Instituto Federal de Minas Gerais \\ ${ }^{2}$ Escola de Música - Universidade Federal de Minas Gerais \\ aluizioneto@ufmg.br, mauricioalvesloureiro@ufmg.br
}

\begin{abstract}
This paper presents a proposal for the use of computational tools for the acoustical analysis of recorded musical sound content in teaching and learning environments as a support for the technical and perceptive development in music performance. The methodology was tested by means of a research carried out during two semesters with an undergraduate class of clarinet students. We describe the implementation of the proposed "augmented study room" as a university level music learning tool.
\end{abstract}

Resumo. Este artigo apresenta uma proposta de aplicação de ferramentas computacionais para análise acústica de conteúdo sonoro musical em ambientes de ensino e aprendizagem, como suporte para o desenvolvimento técnico e perceptivo na performance musical. A metodologia foi testada por meio de uma pesquisa realizada durante dois semestres com uma turma de estudantes de bacharelado em clarineta. Descrevemos a implementação de uma "sala de estudos aumentada” como ferramenta auxiliar no ensino musical de nível superior.

\section{Introdução}

As Tecnologias da Informação e Comunicação (TIC) tiveram um papel crucial no direcionamento das transformações socioculturais ocorridas na segunda metade do século XX. O crescimento da capacidade de processamento dos computadores e redução de seu custo, em conjunto com o nascimento e popularização da Internet, mudaram profundamente a forma como as sociedades se comunicam, compartilham informação e criam conhecimento. As duas últimas décadas foram marcadas pela crescente difusão das redes sociais digitais. A criação e compartilhamento de conteúdo multimídia passou a estar disponível a qualquer pessoa com acesso a um computador pessoal ou smartphone. Atividades ligadas à musica não foram exceção, sofrendo também intenso impacto e profunda transformação. A mudança mais aparente neste contexto é, provavelmente, expressa pela impressionante quantidade de conteúdo musical dos mais diversos gêneros disponível atualmente na Internet. Outro efeito de grande relevância trata da acessibilidade a ferramentas para produção de conteúdo. Mesmo um aparelho de telefone celular de gama intermediária ou um computador pessoal básico podem ser utilizados para produzir materiais que, poucos anos atrás, exigiriam um completo estúdio de gravação com grande quantidade de equipamentos custosos e de acesso muito restrito. $\mathrm{O}$ treinamento e a formação musicais também sofreram influência de tal transformação. Plataformas online permitem 
acesso a um gigantesco e crescente número de gravações de performances de uma mesma peça, possibilitando que intérpretes de todas as partes do globo incorporem as mais diversas referências. Simultaneamente, músicos podem compartilhar seu trabalho e receber feedback externo de forma rápida e universal.

Este artigo trata da investigação de potenciais aplicações de tais avanços tecnológicos no contexto da formação musical de nível superior, em especial quando combinados às tecnologias de Recuperação de Informação Musical, ou MIR, do acrônimo em inglês. ${ }^{1}$. Com foco em aspectos ligados ao desenvolvimento da expressividade musical por meio da incorporação de referências externas $\left(\right.$ modelagem $^{2}$ ) e feedback extrínseco ${ }^{3}$ no processo de aprendizado, apresentaremos resultados obtidos através da um estudo realizado durante dois semestres letivos com uma turma de bacharelado em clarineta. Duas disciplinas foram ofertadas, com foco no desenvolvimento de técnicas associadas às habilidades expressivas na prática do instrumento. Os encontros semanais entre estudantes e mestre foram registrados em áudio. Ferramentas de análise e recuperação de informação musical foram apresentadas e gradualmente introduzidas no processo de aprendizagem. Tais ferramentas oferecem um feedback objetivo sobre características do som produzido, como variações de andamento, timbre, intensidade, afinação, etc., podendo ser utilizadas como referência externa e comparativa entre diferentes execuções de um mesmo excerto.

A motivação para este projeto reside no potencial uso da tecnologia para o desenvolvimento de ambientes de aprendizagem complementares aos métodos tradicionais de ensino de música e no conceito da sala de estudos aumentada. Tais sistemas podem ter implicações em muitos aspectos da prática musical, na redução de custos e no aumento da acessibilidade da formação musical, assim como na ampliação do acesso à modelagem como ferramenta de aprendizado e desenvolvimento da expressividade musical. Este projeto trata do impacto da mediação tecnológica no ensino e aprendizagem musical de nível superior e do uso de feedback aumentado no desenvolvimento da percepção, habilidades técnicas e expressivas no contexto da performance musical. Foram desenvolvidas ferramentas de software para feedback aumentado na prática musical e realizados estudos observacionais sobre o uso das mesmas.

\section{Fundamentação teórica}

Apresentamos a seguir alguns conceitos fundamentais para este projeto.

\subsection{Metacognição}

A metacognição pode ser considerada como a consciência sobre os próprios processos cognitivos, as habilidades que desenvolvemos durante o curso da nossa vida. Está associada à nossa capacidade de deliberar e desenvolver estratégias para resolver problemas relacionados a como esses processos ocorrem. [Flavell 1979] descreve o conhecimento metacognitivo como consistindo "primariamente de conhecimento ou crenças sobre quais fatores ou variáveis atuam e interagem de maneira a afetar o curso e o resultado

\footnotetext{
${ }^{1}$ MIR: Music Information Retrieval. Campo de investigação que trata da extração de informação de áudio musical em formato digital.

2“A modelagem no âmbito musical é o processo de aprendizagem pelo qual o estudante escuta interpretações que lhe servem de modelo, procura imitar, absorve ou replica elementos interpretativos e, eventualmente, transcende essa fase transformando o que absorveu em ideias interpretativas próprias" [Freitas 2013] apud [Gerling and Freitas 2016].

${ }^{3} \mathrm{O}$ "feedback extrínseco" ou "feedback aumentado" trata da informação retornada pelo meio externo sobre uma tarefa executada por um sujeito.
} 
de empreendimentos cognitivos"4. Para [Marzano 1988], "em termos mais simples, a metacognição trata de estar ciente do nosso pensamento à medida que realizamos tarefas específicas, e depois usar tal consciência para controlar o que estamos fazendo"5. No processo de aprendizagem, refinamento e aperfeiçoamento da manipulação de recursos expressivos na construção de uma performance, é importante que o músico tenha uma fonte de feedback que auxilie seu processo metacognitivo. A proposta deste estudo é construir uma estrutura para o uso de ferramentas capazes de fornecer feedback sobre características acústicas da performance. Desenvolvendo e avaliando o uso de sistemas para tal, propomos formas de informar músicos em formação sobre aspectos técnicos de sua performance, dos quais podem ainda não ter plena consciência na etapa que se encontram no processo de aprendizado. Tais recursos podem apoiar o desenvolvimento de suas habilidades metacognitivas, contribuindo assim para o refinamento da percepção, consciência e controle de recursos expressivos (tais como variações de andamento, articulações, legatos, e outros).

\subsection{Feedback no processo de aprendizado}

Um dos aspectos essenciais da prática compreende as informações que um instrumentista recebe sobre os resultados das ações realizadas. Este feedback é em algum nível intrínseco a toda e qualquer atividade que envolva o corpo humano, uma vez que os músculos e nervos sempre retornarão alguma forma de informação sobre as ações executadas para o sistema nervoso central. Isto é especialmente verdadeiro quando movimento e tato estão envolvidos. [Schmidt and Lee 1988] caracteriza o conceito de feedback em duas classes: Feedback Intrínseco: inerente à mera realização da tarefa em questão. É fornecido por meio dos órgãos sensoriais do próprio sujeito que a executa. Em instrumentos musicais acústicos, feedback táctil é inerente à produção sonora, o que o torna essencial no desenvolvimento das habilidades musicais necessárias para controlar aspectos acústicos da performance [Birnbaum and Wanderley 2007].

Feedback extrínseco: Também denominado "Feedback Aumentado", incorpora informações oriundas de fontes externas. Ocorre em situações nas quais algum dado relativo à tarefa executada é enviado de volta ao executante, como ofeedback verbal fornecido por um professor para um aluno ou um indicador luminoso na operação de algum equipamento eletromecânico. O feedback aumentado apresenta informações que não estariam prontamente disponíveis pela mera execução da tarefa.

\subsection{MIR: Recuperação de Informação Musical}

Graças a recentes avanços em ferramentas para processamento digital de sinais, tornou-se possível entender, modelar e sintetizar elementos das habilidades musicais em sistemas computacionais. Tais sistemas permitem a recuperação automática de informações de gravações de uma performance, sendo capazes de descrever aspectos sutis de modulações de parâmetros acústicos e, portanto, efeitos da intenção expressiva do executante. $\mathrm{O}$ MIR é um efervescente campo de pesquisa que segue em franco desenvolvimento [Schedl et al. 2014, Downie 2003]. Interdisciplinar por natureza, combina disciplinas

\footnotetext{
4"Metacognitive knowledge consists primarily of knowledge or beliefs about what factors or variables act and interact in what ways to affect the course and outcome of cognitive enterprises."

5 "In simpler terms, metacognition is being aware of our thinking as we perform specific tasks and then using this awareness to control what we are doing."
} 
como processamento de sinais, reconhecimento de padrões, aprendizagem de máquina, psicoacústica, percepção musical e cognição e musicologia. Sua presença na indústria musical ganhou força com o crescimento do modelo de mercado baseado no streaming. Algoritmos para MIR visam permitir que computadores "escutem" ou "interpretem" dados a partir do áudio. Suas técnicas buscam preencher a lacuna semântica entre áudio e descrição, permitindo a extração automática de informações de alto nível de música armazenada em formatos digitais como MP3, CDs, DVDs, etc.. Um conjunto de ferramentas para Recuperação de Informação Musical compõe a base da tecnologia utilizada neste projeto.

\section{Metodologia}

Durante dois semestres letivos os estudantes tiveram suas aulas gravadas em áudio, incluindo diálogos entre alunos, mestres e profissionais convidados. Em situações pontuais foram feitos também registros em vídeo. As disciplinas ofertadas contaram também com a participação de um professor do instrumento e um pesquisador/facilitador para introduzir o acesso ao uso das novas tecnologias apresentadas e realizar as gravações de forma sistemática e controlada. Professores de outras instituições foram convidados a participar pontualmente. Os encontros contaram com duas abordagens complementares: na primeira os alunos trabalhavam com o mestre da forma que o faziam tradicionalmente, na qual peças do repertório eram apresentadas, executadas e discutidas pela classe. Através de metáforas, indicações sonoras e gestuais e a partir de repetições e demonstrações dos trechos de maior complexidade, o professor orientava seus pupilos em correções de equívocos técnicos ou interpretativos. A segunda parte tratava da introdução às tecnologias para análise computacional da performance, onde os alunos tinham contato com as ferramentas e práticas ligadas ao uso de gravações, processamento de áudio e extração de informação musical no desenvolvimento de seu aprendizado. Nas próximas seções descreveremos de forma detalhada a estrutura, hardware, softwares e sistemas utilizados ao longo das disciplinas.

\subsection{Arquitetura proposta - Um sistema modular e livre}

Visando a futura incorporação das abordagens propostas neste trabalho a outras plataformas já existentes e em desenvolvimento, foi realizado o desenho de uma arquitetura de software e hardware flexível e expansível, adaptável a diferentes situações e recursos.

A proposta desenvolvida se inspira em padrões da arquitetura de Software, tais como o MVC (Model-View-Controller - ver [Krasner et al. 1988]), e o suporte a plugins, e foi implementada utilizando exclusivamente software livre. Consideramos um sistema composto por três blocos que podem ser preenchidos por diferentes implementações de funcionalidades semelhantes. São eles:

- Aquisição de dados: Utilizamos os sistemas disponíveis em laboratório para captura de áudio, vídeo e movimento. Também foram utilizados plugins compatíveis com o padrão $V A M P$ e scripts nas linguagens Python e R para processamento do sinal adquirido, tratamento dos dados e extração de informação. Este bloco poderia ser alimentado por conteúdos oriundos de outras fontes, tais como gravações de áudio e vídeo disponibilizadas online, CDs, DVDs, partituras em formato MusicXML ou MIDI, etc.;

- Armazenamento / banco de dados: foi criado um banco SQL com ponteiros para arquivos armazenados em um servidor montado em nosso laboratório. Arquivos de 
áudio, vídeo e texto foram armazenados e indexados através de tabelas SQL, permitindo inserção, busca e recuperação de informação através de uma plataforma web disponibilizada para acesso remoto pelos alunos e professores do curso.

- Visualização e análise: foi utilizado o software Sonic Visualiser e plugins VAMP para ler arquivos de áudio e anotações em formato CSV, exibindo-as de forma amigável.

\subsection{Estrutura física e hardware utilizados}

As aulas foram realizadas em uma sala com tratamento e isolamento acústico básicos, equipada com microfone, interface de áudio, câmera de vídeo e um computador pessoal. O servidor responsável pelo armazenamento e processamento das informações capturadas se localizava em uma sala separada e com isolamento acústico, de forma evitar a inserção de ruídos oriundos de dispositivos como ventoinhas e fontes de tensão nas gravações. Um gravador portátil Zoom $\mathrm{H} 4 \mathrm{n}$ foi utilizado para registrar diálogos, entrevistas e demonstrações durante as sessões. As performances realizadas pelos alunos e mestres foram capturadas por um microfone conectado ao servidor através de uma placa de som (Conversor $\mathrm{A} / \mathrm{D}^{6}$ ). Este trabalho visa apresentar recursos a serem explorados em contextos de aprendizagem musical dentro e fora de um laboratório. Buscamos investigar tecnologias que possam ser utilizadas por estudantes e professores que não disponham de equipamentos de gama avançada como aqueles encontrados em estúdios de gravação ou centros de pesquisa. Microfones e interfaces de áudio de gama não-profissional integradas em notebooks e celulares foram testadas para efeito de comparação. Não foram detectas perdas de qualidade que impossibilitassem as abordagens aqui propostas, apesar de apresentarem uma menor confiabilidade com relação à precisão das medições realizadas. A maioria dos dispositivos oferecidos para o consumidor final no mercado trabalha com taxas de amostragem entre $44.100 \mathrm{~Hz}$ e $96 \mathrm{kHz}$, e quantização de 16 a $24 \mathrm{bits}$. Tal amostragem e resolução são suficientes para a maioria das aplicações de áudio.

\subsection{Processamento Digital de Sinais e Extração de Informação Musical}

Para aquisição, visualização e processamento de dados foi utilizado um computador rodando o sistema Debian GNU/Linux. Todo o material de áudio gravado em aula foi salvo e indexado em uma base de dados, ficando disponível para reprodução, análise e processamento com o uso das ferramentas selecionadas. Os softwares Audacity e Sonic Visualiser, utilizados neste processo, implementam suporte a uma arquitetura aberta para desenvolvimento de plugins denominada Vamp. Graças a tal arquitetura funções muito específicas e complexas podem ser criadas e implementadas com facilidade, utilizando os recursos técnicos das plataformas compatíveis já existentes. No caso da nossa disciplina utilizamos uma série de plugins específicos para a segmentação, análise e extração de informação de áudio musical. As informações extraídas podem ser visualizadas de formas variadas, facilitando sua interpretação. Nesta seção listamos e descrevemos os pacotes utilizados para realizar a segmentação e visualização dos parâmetros acústicos manipulados pelos músicos em suas performances.

\footnotetext{
${ }^{6}$ Os conversores A/D-D/A são os sistemas principais das interfaces de áudio (ou "placas de som") presentes na maioria dos computadores fabricados a partir de meados da década de noventa. São os elementos responsáveis por converter o sinal analógico oriundo de um microfone ou outro sistema de transdução em informação digital, armazenável e tratável em computadores digitais.
} 
IX Congresso Brasileiro de Informática na Educação (CBIE 2020)

Anais do XXXI Simpósio Brasileiro de Informática na Educação (SBIE 2020)

\subsubsection{Expan Plugin}

Implementação dos algoritmos desenvolvidos e implementados no Expan Toolbox [Campolina et al. 2009], um pacote desenvolvido com a intenção de facilitar a análise de um conjunto de parâmetros acústicos ligados à expressividade musical.

\subsection{2. pYIN}

Implementação do YIN, um tradicional algoritmo para detecção de frequência fundamental (pitch) de sinais monofônicos. Desenvolvido por Matthias Mauch na Queen Mary University of London, permite observar variações sutis de afinação, possibilitando a verificação de erros recorrentes, além de manipulações intencionais realizadas como recurso expressivo em situações onde isso é desejável [Mauch and Dixon 2014].

\subsubsection{BBC Vamp plugin collection}

Desenvolvida pelo BBC Research and Development, esta coleção de plugins permite a extração de vários parâmetros acústicos associados às manipulações sonoras realizadas por músicos. Foi escolhido por ter implementações muito eficientes, acessíveis e de fácil utilização, além de ser um pacote livre e de código aberto disponível para download no site de seu desenvolvedor. As ferramentas utilizadas foram: Mean Energy Estimation, Harmonic Spectral Centroid e Pitch Class Profile.

\subsection{Processamento de dados e estatística}

Performances gravadas e processadas com o uso das ferramentas descritas acima podem ser analisadas de forma simples e intuitiva através de representações gráficas permitidas por softwares tais como Sonic Visualiser e Audacity. No entanto, quando o volume de dados é muito grande (como no caso de gravações de séries de ensaios, práticas e/ou aulas semanais geradas ao longo de meses seguidos) é desejável a possibilidade de se extrair informação referente a relação entre um grande número de execuções. Um exemplo deste tipo de situação é a investigação sobre a evolução de tais práticas ao longo do tempo, ou mesmo uma avaliação da consistência das performances em condições distintas. Diversas questões podem ser respondidas por tal base massiva de dados, tais como relações entre características interpretativas e estados emocionais de um determinado músico, influências de uma determinada abordagem pedagógica no desenvolvimento de um grupo de estudantes, etc. Tais análises podem ser realizadas por meio de ferramentas tradicionais para análise estatística ou técnicas oriundas da inteligência artificial, área em franco desenvolvimento e com grandes potenciais no contexto do ensino musical.

\section{Análise e Resultados}

As disciplinas ofertadas como parte deste estudo tiveram como foco o desenvolvimento de aspectos da performance relativos às manipulações sonoras de caráter expressivo realizadas pelos músicos. Foram selecionados para análise neste artigo: Manipulação temporal (Ralentandos e acelerandos); Articulação (Legato, stacato, perfil do ataque); Manipulação Dinâmica (Crescendo, decrescendo) e Acoplamento em Performance de 
Conjunto. Foram extraídos descritores acústicos para caracterizar tais aspectos a partir do áudio gravado, assim como métodos de visualização dos mesmos. Essas ferramentas foram utilizadas em sala de aula pelos alunos e professores.

\subsection{Manipulações Temporais}

Diversos trechos explorados no programa das disciplinas apresentam indicações de variações expressivas de andamento indicadas na partitura. O trecho selecionado para a análise a seguir trata de uma cadência e permite grande liberdade ao intérprete. $\mathrm{O}$ perfil das curvas de acelerando e ralentando executado por músicos profissionais e sua relação com a expressividade e individualidade musical é um tema amplamente explorado na literatura. Por meio da segmentação do sinal sonoro a partir da identificação do instante do ataque e início das notas, podemos extrair os valores de Intervalo Entre Onsets (IOI) - tempo decorrido entre ataques de notas subsequentes. Tal informação nos fornece um valor relativo a um "andamento local" quando normalizado em função da duração da figura rítmica expressa na partitura. A variação do mesmo ao longo de uma frase pode ser representada por uma curva de IOIs, indicando o perfil de variação temporal realizado pelo intérprete (Figura 2).

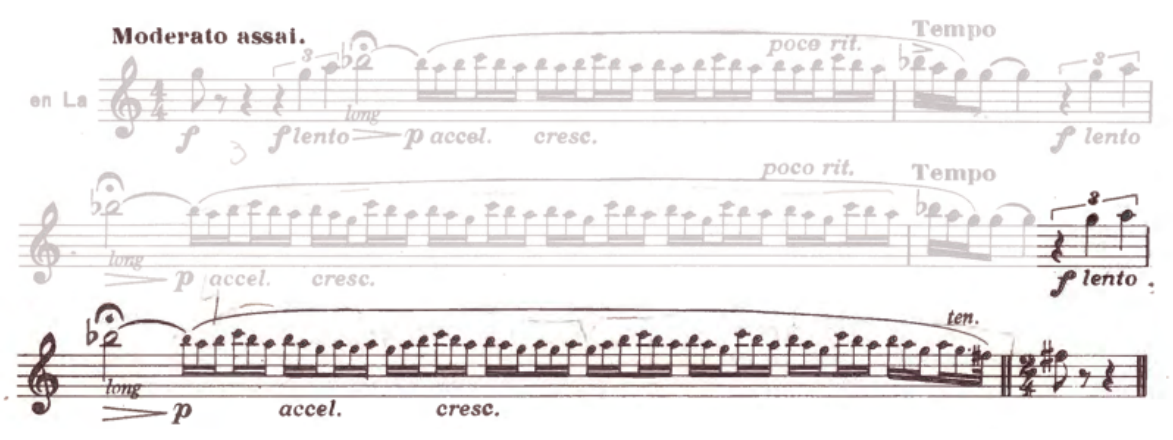

Figura 1. Excerto da Suíte Sinfônica Scheherazade, de Nicolai Rimsky-Korsakov

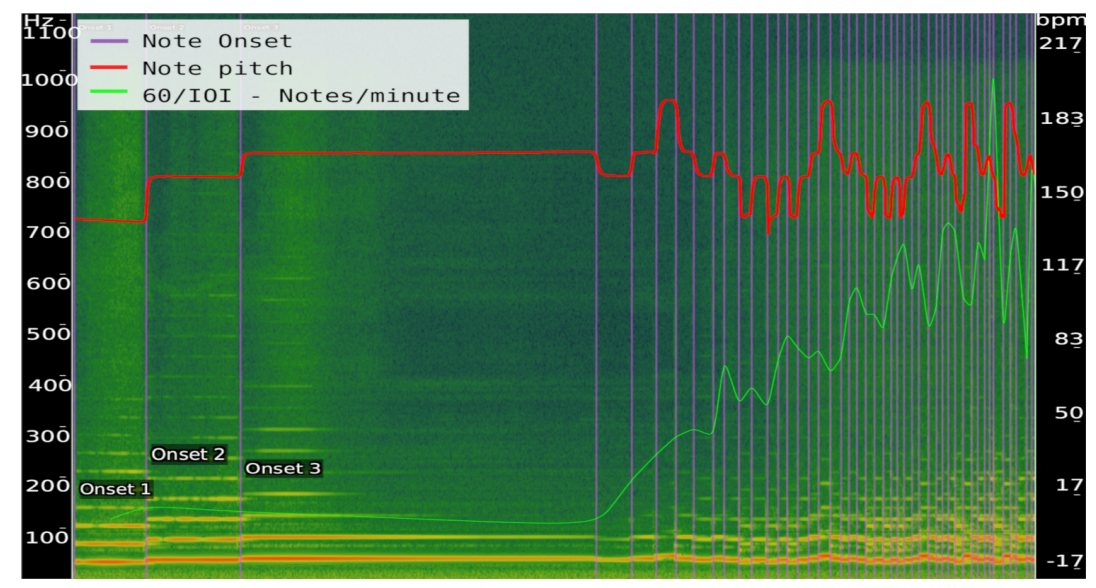

Figura 2. Análise da curva de acelerando da execução do excerto da Figura 1

\subsection{Articulação}

As figuras 4 e 5 exibem análises realizadas a partir da gravação de duas interpretações que permitem comparar dois estilos distintos de articulação de notas adotadas por 2 clarinetistas profissionais na interpretação de um excerto extraído do segundo movimento 
(Andante molto moto) da Sinfonia No. 6 em Dó Maior op. 68, de Ludwig Van Beethoven (Figura 3). As regiões marcadas em diferentes tons de cinza indicam as durações de cada uma das sete notas destacadas na partirura, delimitadas pelos IOI's. A largura dos retângulos revelam os alongamentos e encurtamentos das durações de cada nota manipuladas pelos músicos em sua interpretação. A curva cinza representa as alturas das notas e a negra a evolução temporal da intensidade sonora. Comparando as duas execuções, podemos observar que as notas da primeira execução são mais curtas e que há quedas mais abruptas de energia entre elas, o que indica serem mais articuladas nesta interpretação. A segunda execução exibe uma curva de energia mais estável e em um nível médio mais elevado, com menor variação de energia entre uma nota e outra, indicando que as notas nesta interpretação são menos articuladas, mais ligadas.

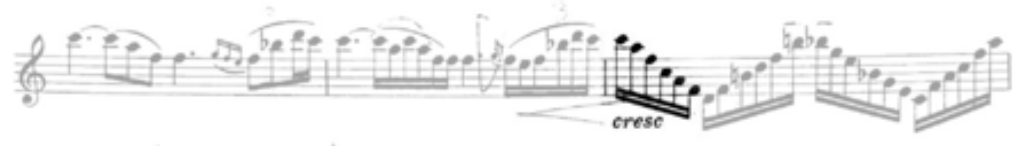

Figura 3. Excerto da Sinfonia No 6 em Dó Maior op. 68, de Ludwig Van Beethoven

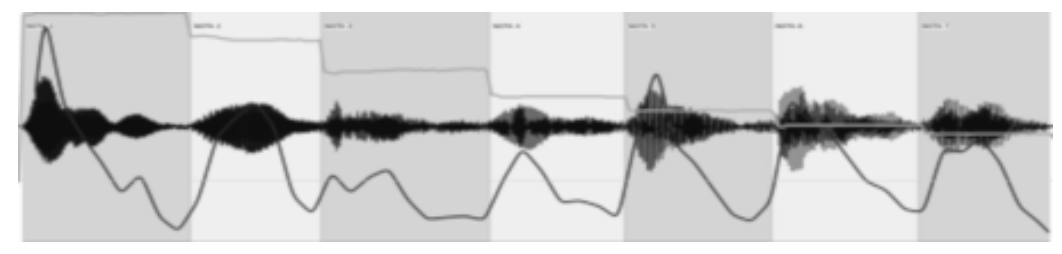

Figura 4. Execução realizando articulações breves e desligadas

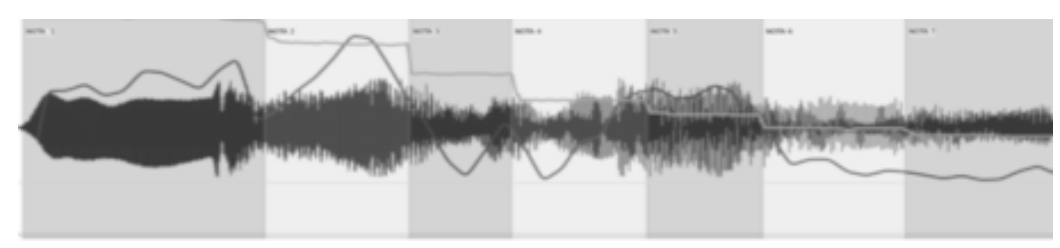

Figura 5. Execução realizando articulações longas e de caráter legato

\subsection{Performance de Conjunto - Acoplamento}

Durante a disciplina intitulada "Duos, Trios e Quartetos para Clarineta e Fagote" foram trabalhadas peças para formações em grupo, permitindo que investigássemos o uso do sistema neste contexto. Como exemplo ilustrativo do tipo de informação que foi possível obter a partir da abordagem proposta, selecionamos um trecho da peça Divertimento Nr.3, KV 439b., de Wolfgang Amadeus Mozart. Neste trecho, o mestre fazia indicações de andamento regendo os alunos, além do estalar de seus dedos. Realizando uma análise informada pelo contexto e filtragem do sinal de áudio estéreo obtido pelo gravador, foi possível extrair o tempo indicado pelo mestre, assim como os instantes de ataque das notas executadas pela primeira clarineta e fagote. Na figura 7 apresentamos uma captura de tela do espectrograma de um trecho da sessão em questão, anotado com os pontos de onset extraídos do sinal de áudio marcados como linhas verticais, tanto para mestre quanto para a primeira clarineta, e as curvas de andamento. Neste exemplo específico utilizamos, além dos plugins VAMP descritos acima, alguns algoritmos desenvolvidos na 
linguagem $\mathrm{R}$ para processar os dados extraídos do áudio e inserir o resultado novamente no programa Sonic Visualiser para visualização.

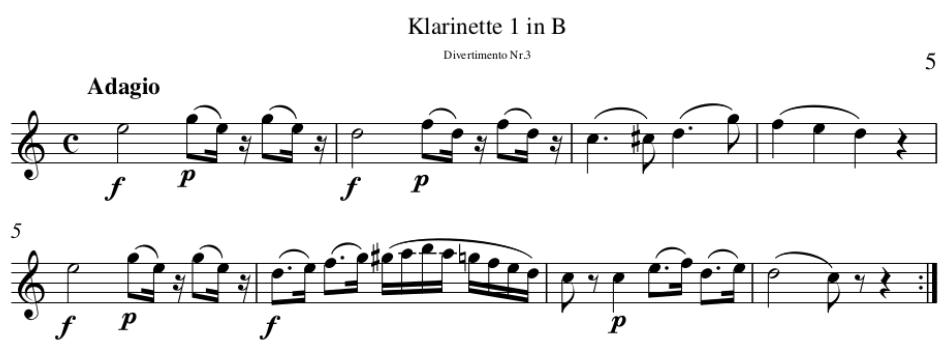

Figura 6. Excerto do Divertimento Nr. 3 para 2 Clarinetas e Fagote, de W. A. Mozart, KV 439b.

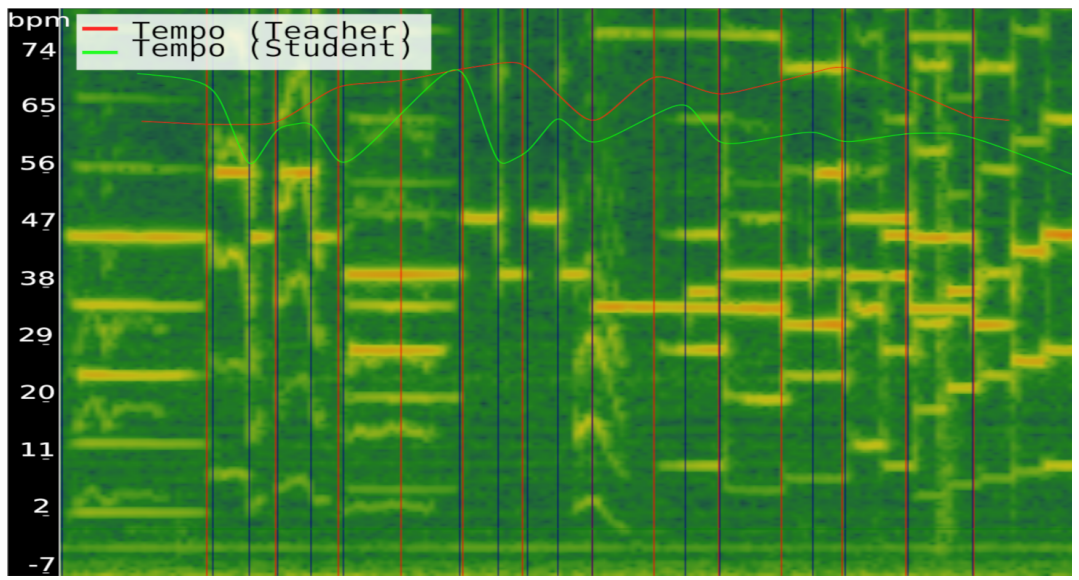

Figura 7. Andamento regido pelo professor (em vermelho) e realizado pelo aluno (em verde) do trecho apresentado na Figura 6

\section{Conclusão}

Ao contrastar relatos dos sujeitos que participaram do experimento com as análises acústicas realizadas, constatamos que parte de suas percepções sobre qualidades sonoras das performances podem ser extraídas do áudio gravado de forma procedural. Gravações em estéreo, material de vídeo, Motion Capture e acesso às partituras dos trechos analisados facilitam a automatização desse processo. Estudantes relataram que a utilização de material audiovisual de referência em conjunto com feedback aumentado da própria performance contribuem no processo de modelagem. As ferramentas apresentadas otimizam esse processo ao permitirem a comparação automática entre referências externas e a performance do aluno que utiliza o sistema. Referências e feedback externos oriundo de fontes diversas foram considerados o ponto mais positivo das disciplinas experimentais criadas de acordo com o relato dos alunos. Através das metodologias aqui propostas, demonstramos ser possível incorporar referências oriundas de gravações multimodais disponíveis em formato digital para criar material de feedback extrínseco e fonte de referência externa. Tal abordagem amplia o acesso a esse tipo de processo de forma proporcional a crescente oferta de conteúdo livremente disponível em plataformas digitais. O uso de técnicas avançadas de MIR para feedback aumentado em um cenário de ensino e 
aprendizagem musical ainda não foi profundamente explorado. Há uma grande variedade de oportunidades de pesquisa e desafios no campo e direções promissoras estão sendo constantemente descobertas. Consideramos que este trabalho é uma dessas iniciativas, sendo que parte de nossas hipóteses foi confirmada pelos dados experimentais adquiridos e relatos dos sujeitos participantes. Também esperamos que isso possa servir como inspiração para a pesquisa que está por vir e que nossos experimentos possam vir a ser postos a prova e replicado por outros.

\section{Referências}

Birnbaum, D. M. and Wanderley, M. M. (2007). A systematic approach to musical vibrotactile feedback. Proceeding of the International Computer Music Conference, pages 397-404.

Campolina, T., Loureiro, M., and Mota, D. (2009). Expan: a tool for musical expressiveness analysis. Proceedings of the 2nd International Conference of Students of Systematic Musicology, pages 24-27.

Downie, J. S. (2003). Music information retrieval. Annual review of information science and technology, 37(1):295-340.

Flavell, J. H. (1979). Metacognition and Cognitive Monitoring: A New Area of CognitiveDevelopmental Inquiry. American Psychologist, 34(10):906-911.

Freitas, S. G. A. d. (2013). Modelagem como estratégia para o desenvolvimento de recursos expressivos na performance pianistica: três estudos de casos.

Gerling, C. C. and Freitas, S. (2016). Reflexões sobre a modelagem como estratégia de estudo: relatos de duas investigações com estudantes de graduação e pós-graduação. Arteriais-Revista do Programa de Pós-Gradução em Artes, 1(1):90-106.

Krasner, G. E., Pope, S. T., et al. (1988). A description of the model-view-controller user interface paradigm in the smalltalk-80 system. Journal of object oriented programming, 1(3):26-49.

Loureiro, M., Magalhães, T., et al. (2008). Segmentação e extração de descritores de expressividade em sinais musicais monofônicos. Seminário Música Ciência Tecnologia, $1(3)$.

Marzano, R. J. e. a. (1988). Dimensions of thinking: A framework for curriculum and instruction. ERIC.

Mauch, M. and Dixon, S. (2014). pyin: A fundamental frequency estimator using probabilistic threshold distributions. In 2014 IEEE International Conference on Acoustics, Speech and Signal Processing (ICASSP), pages 659-663. IEEE.

Schedl, M., Gómez, E., Urbano, J., et al. (2014). Music information retrieval: Recent developments and applications. Foundations and Trends $\mathrm{R}$ in Information Retrieval, $8(2-3): 127-261$.

Schmidt, R. A. and Lee, T. (1988). Motor control and learning. Human kinetics. 